\title{
Videofluoroscopy in Macular Disease
}

\author{
S. T. D. ROXBURGH and W. M. HAINING
}

Dundee

\begin{abstract}
Summary
Fluorescein Angiography has contributed immensely to our understanding and management of macular disease. Ridley ${ }^{1}$ first introduced the concept of television ophthalmoscopy and in 1973 Van Heuven and Schaffer ${ }^{2}$ reported the value of videofluoroscopy using a low light level system and an image intensifier. Major advances in electronic imaging technology since then have permitted high quality videofluoroscopy to be developed ${ }^{3}$ in particular the integral silicone intensified tube (SIT) allowed ultra low light levels to be recorded.

A videofluorography system has been in routine use in our department for the last six years and we have found it to have certain advantages over conventional photography in macular disease. In this presentation we demonstrate some of these.
\end{abstract}

Our present system (Fig. 1) links the stereoophthalmoscope 110 from V. E. B. Carl Zeiss Jena GDR via a beam splitter with a specially designed adaptor to a low light level monochrome television camera (Badger 25 SIT).

The advantages of videofluoroscopy over conventional photographic recording are listed below (Table I).

(1) The whole dye transit is recorded. The complete fluorescein run is shown. Dye entry is never missed and the real-time dynamic aspects of the transit can be fully appreciated. Abnormal or delayed vascular filling and the evolution of extravascular fluorescence are vividly demonstrated. The motility of certain lesions is seen. This is of particular value in pulsatile lesions and the movement of emboli in the retinal vascular tree may also be recorded.

(2) Immediate result. The angiogram may be observed simultaneously. There is no delay due to photographic processing allowing immediate management decisions and laser treatment.

(3) Cost saving. Considerable saving in the cost of photographic materials and technicians' time may be made. Videotapes are relatively cheap and may subsequently be edited and re-used.

(4) Patient acceptability. The silent low light level system reduces patient discomfort stress during the angiogram. The reduced light level may also have implications regarding light toxicity in already compromised maculae.

(5) Simple to operate. The system may be operated with minimal instruction without the need of skilled technical assistance.

(6) Quantitative analysis of the image. Computerised digital analysis of the electronic image is possible and allows blood flow parameters to be measured. ${ }^{4}$

The disadvantages of the system are the loss of resolution compared with conventional photography, and the inability to have a permanent record in the patient's file. The resolution is about a quarter of that achieved by high speed photographic film. ${ }^{2}$

The loss of resolution has not proven a practical problem. Although capillary detail is not 




Fig. 1. Video camera set up showing Badger SIT camera mounted on television ophthalmoscope with videorecorder set at stop frame.

as good as that seen with photographic techniques the video film is adequate for clinical purposes.

With regard to macular disease, videofluoroscopy has two main applications; diagnostic and therapeutic.

\section{Diagnostic}

The dynamic display allows pulsatile lesions to be demonstrated. Figure 2 shows a hypofluorescent macular lesion in a patient with the maculopathy of ageing. On funduscopy the lesion appeared to be a small subretinal haemorrhage. Throughout the fluorescein angiogram the lesion was pulsatile and remained densely hypofluorescent, blocking underlying choroidal fluorescence. We believe such a lesion has not previously been described and its nature can only be surmised. It appears to be an encapsulated haemorrhage, the surrounding normal choroid giving rise to the pulsation. Subretinal neovascularisation subsequently appeared at the site of the haemorrhage. The pulsatile nature of the lesion would not have been appreciated by conventional photography.

The real-time display of extravascularisation of fluorescein allows appreciation of certain macular lesions. For example the case of pigment epithelial rip illustrated in Figure 3 shows very clearly the early marked hyperfluorescence of the bare choroid contrasting with the dark band due to the presumed rolled edge of the pigment epithelium and the late pooling of fluorescein under the pigment epithelial detachment superiorly. These features are uniquely demonstrated by videofluoroscopy.

Table I Advantages and disadvantages of videofluoroscopy

\begin{tabular}{lc}
\hline \multicolumn{1}{c}{ Advantages } & Disadvantages \\
\hline Whole dye transit & Loss of resolution \\
Immediate result & Record not possible in \\
Cost saving & patient's file \\
Patient acceptability & \\
Simple to operate & \\
Quantitative analysis & \\
possible & \\
\hline
\end{tabular}



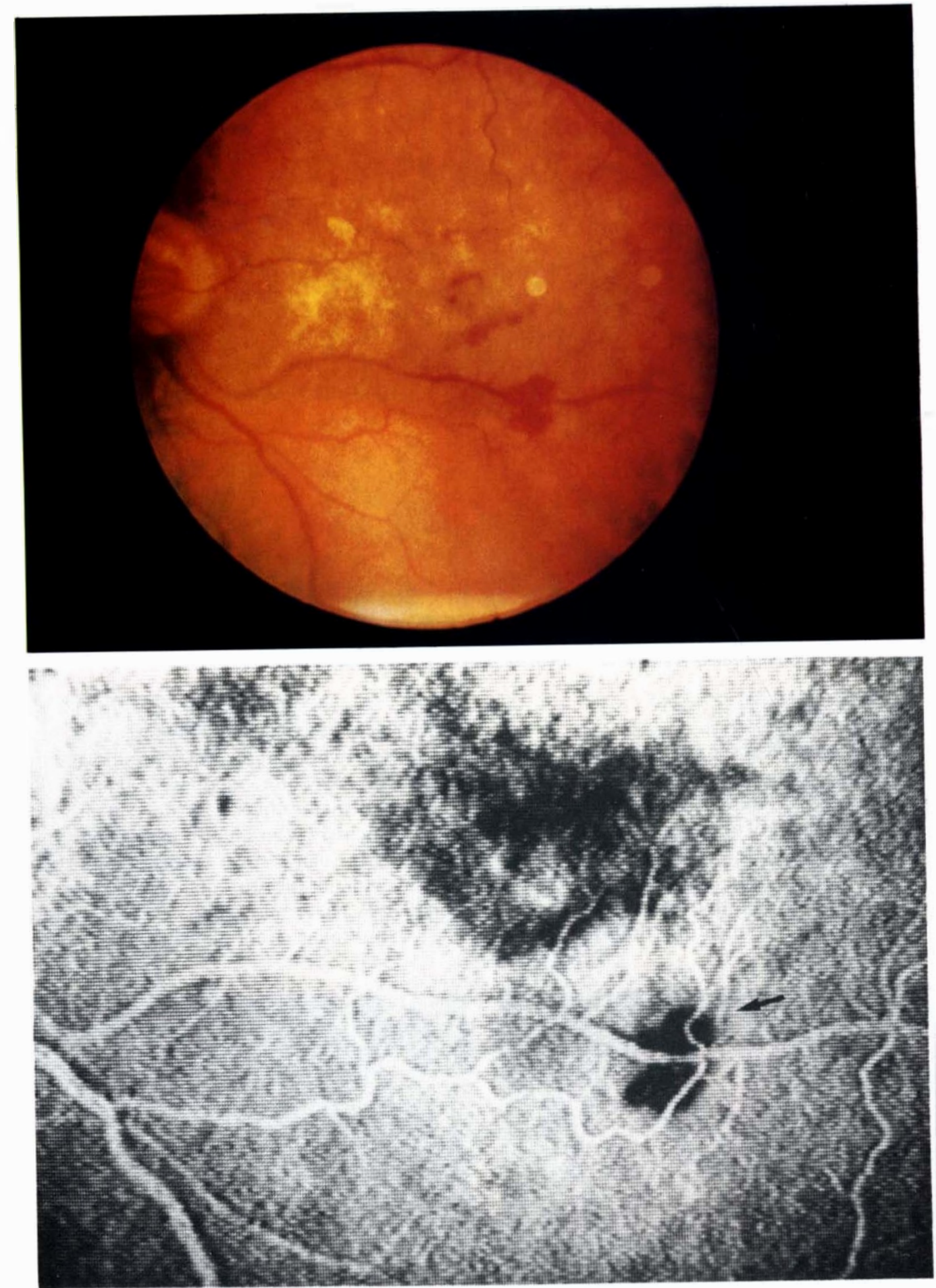

Fig. 2. Fundus photograph (2a) and photograph of the videofluorogram (2b) from a patient with the maculopathy of ageing. The hypo-fluorescent lesion (arrowed) was pulsatile throughout the angiogram and corresponds to the red subretinal lesion seen on fundoscopy. 


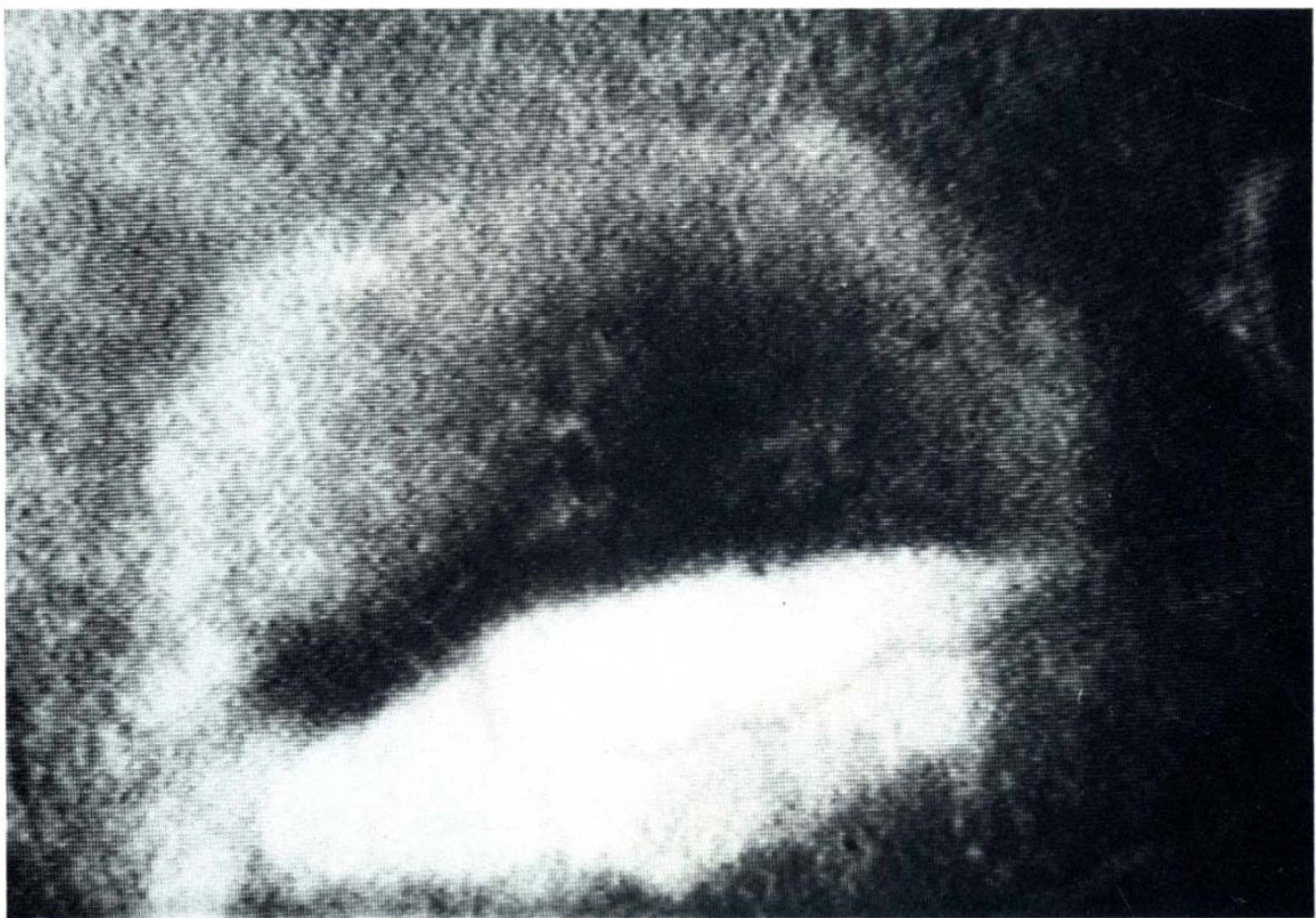

Fig. 3. Photograph from the videofluorogram of a pigment epithelial rip.

\section{Therapeutic}

We find videofluroscopy a valuable aid in delineating areas requiring laser treatment. Argon laser has been proven to be of value in the management of ageing macular degeneration complicated by subretinal neovascular membrane formation out with the fovea.$^{5,6.7}$

Prior to treatment fluorescein angiography is essential in delineating the membrane.
Videofluoroscopy typically demonstrates the lacy or cartwheel appearance of the membrane with early filling and late leakage.

Atypical membranes may present as 'hot spots' which show gradually increasing fluorescence. In such cases if there is supportive clinical evidence of subretinal neovascularisation (SRNV) in terms of lipid or blood around the leaking focus, treatment has been advo-
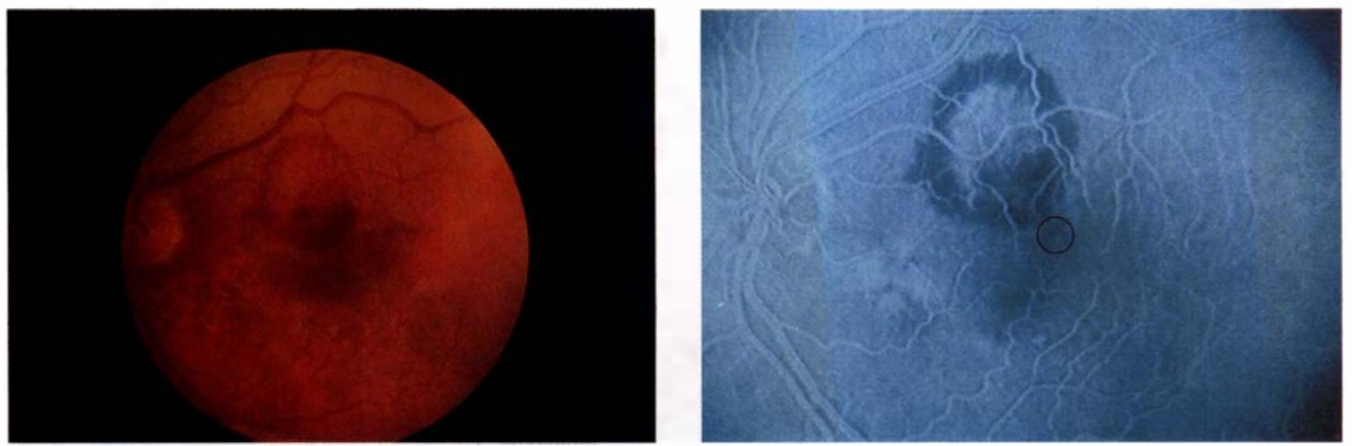

Fig. 4. Fundus photograph (4a) and photograph of a videofluorogram (4b) of a patient with a treatable subretinal neovascularisation complicating the maculopathy of ageing. The central black circle on the fluorogram corresponds to a diameter of $400 \mu$ and allows assessment of treatable lesions. 

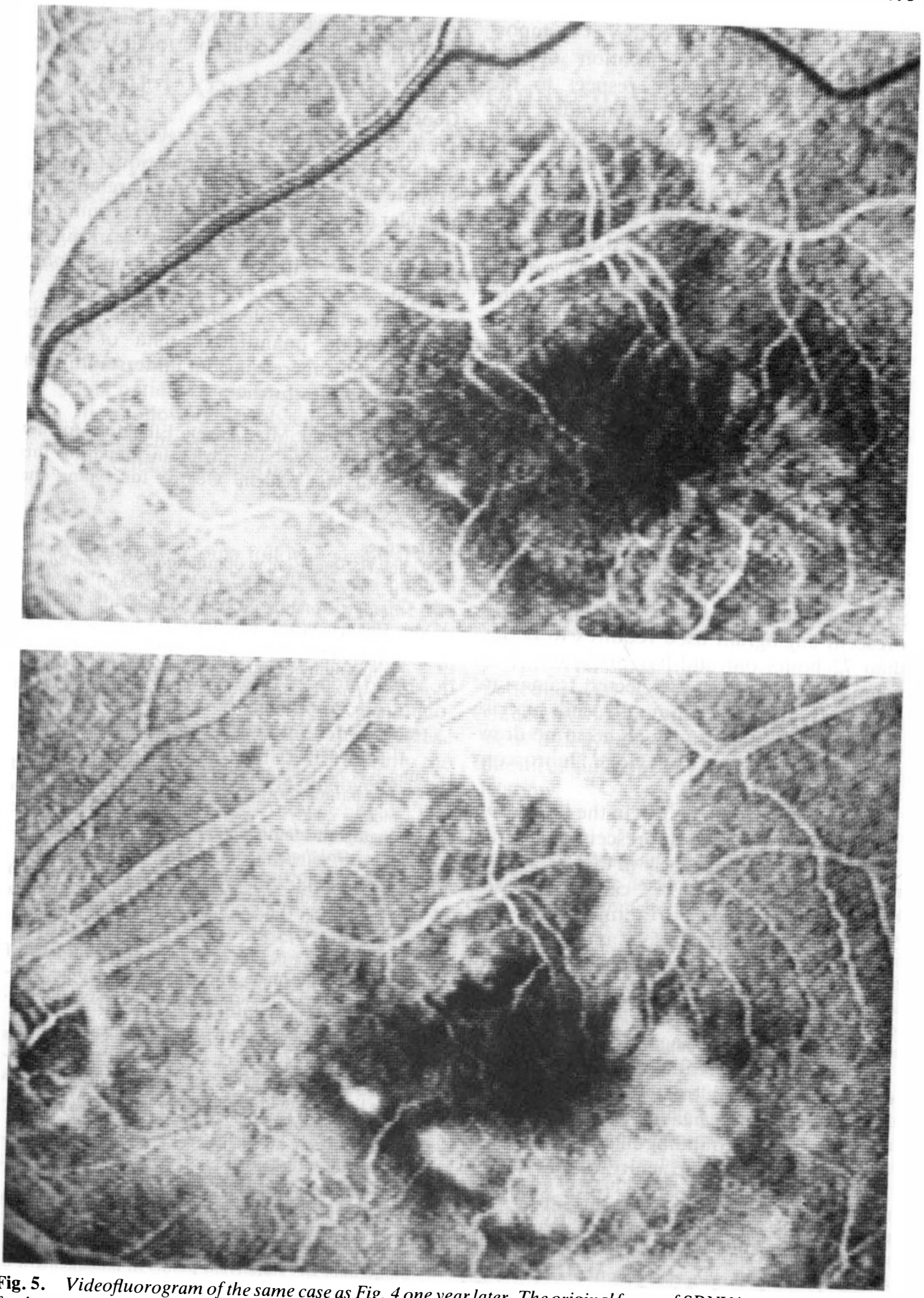

fresh neovascular fan is evident. Figure 5 a is in the early venous phase original focus of SRNV is well treated and a 
cated. ${ }^{8}$ Having diagnosed SRNV it is important to determine the location of the membrane accurately with respect to the fovea since treatment of membranes with argon blue/green laser greater than $200 \mu$ from the centre of the foveal avascular zone is of benefit. ${ }^{5.6}$ To determine the distance of the SRNV from the fovea the Macular Photocoagulation Study Group ${ }^{5}$ used a magnifier with a reticule adaptor and the Moorfields Macular Study Group used a measuring grid based on the optic disc diameter. ${ }^{9}$ Alternatively one may assume the diameter of a major retinal vein to be $170 \mu$ and make calculations accordingly. ${ }^{8}$ We have used a simple circle corresponding to $400 \mu$ based on average readings of retinal diameter on the video screen (Fig. 4). These distances, however, are approximate and subject to great biological variation.

The circle can be centred over the darkest point on the angiogram corresponding to the foveal avascular zone. Conventionally the fluorescein angiogram used requires to be less than 72 hours old and has preferably been taken on the same day. Selected frames are projected while treating to aid precise localisation. Alternatively an accurate drawing is made. The use of video fluoroscopy simplifies management.

The videotape can be run in the laser room immediately after the fluorescein angiogram and set at stop frame early in the dye transit. The neovascular complex can be precisely located with reference to retinal vessels and other visible landmarks and treatment applied. We use the technique advocated by Singerman. ${ }^{10}$ The neovascular complex is outlined with $100 \mu$ burns and then treated with heavy $200 \mu$ burns. Angiography is repeated two weeks after treatment. Figure 4 shows early frames from a treatable case. The treatment was applied heavily over the leakage area and the surrounding haemorrhage. The patient retained a good acuity for one year but subsequently developed a fresh subfoveal focus. Figure 5 demonstrates clearly the neovascular fan and the previously successfully treated area.

We have found videofluoroscopy a quick and convenient aid in treating macular lesions. We have used SRNV to illustrate the technique but it is applicable to all treatable macular disease.

\section{Conclusion}

We have found our present system to have certain advantages over conventional photographic fluorescein angiography in relation to macular disease and would highly recommend the system, especially to those working in departments without good photographic back-up.

For the future, electronic image analysis may provide quantitative data of videofluorography, increasing our understanding of macular disease further.

We wish to thank Mrs. Angela Ellingford for her valuable assistance and advice in preparing this paper and Mrs. Allene Gearce for typing the manuscript.

\section{References}

${ }^{1}$ Ridley H: Television in ophthalmology. Proceedings of the 16th International Congress of Ophthalmology. London: 1950; 2: 1397-404.

2 Van Heuven WAS, Schaffer C: Advances in televised fluorescein angiography. International Symposium of Fluorescein Angiography, Tokyo. In: Fluorescein Angiography. Tokyo: Igaku Shoin. 1973; 10-14.

3 Haining WM: Videofunduscopy and fluroscopy. $B r$. J. Ophthalmol. 1981; 65: 702-6.

+ Jung F et al: Quantification of characteristic blood flow parameters in the vessels of the retina with a picture analysis system for video-fluorescence angiograms: initial findings. Graefe's Arch. Clin. Exp. Ophthalmol. 1983; 221: 133-6.

${ }_{5}^{5}$ Macular Photocoagulation Study Group: Argon laser for senile macular degeneration; results of a randomized clinical trial. Arch. Ophthalmol. 1982; 100: 912-8

${ }^{6}$ The Moorfield Macular Study Group: Treatment of senile disciform degeneration: a single-blind randomised trial by argon laser photocoagulation. Br. J. Ophthalmol. 1982; 66: 745-53.

${ }^{7}$ Coscas G, Soubrane G: Photocoagulation des néovaisseaux sousrétiniens dans la degénérescence maculaire sénile par laser á argon; resultats de l’étude randomisée de 60 cas. Bull. Mem. Soc. Fr. Ophtalmol. 1982; 94: 149-54.

${ }^{8}$ Folk JC: Aging macular degeneration: Clinical features of treatable disease. Ophthalmology 1985; 92: 594-602.

${ }^{9}$ Gregor Z, Bird AC, Chisholm IH: Senile disciform macular degeneration in the second eye. Br. J. Ophthalmol. 1977; 61: 141-7.

11 Singerman LJ: Important points in management of patients with choroidal neovascularisation. Ophthalmology 1985; 92: 610-4. 